\title{
2D DOA Estimation Algorithm for Massive MIMO using Propagator Method
}

\author{
Basit Iqbal and Rahim Umar ${ }^{2 *}$ \\ College of Electronic and Information Engineering \\ Nanjing University of Aeronautics and Astronautics, Nanjing 211106, China \\ 1'basitiqbal01@hotmail.com, ${ }^{2}$ rahim_nuaa@hotmail.com
}

\begin{abstract}
In this paper, a 2D propagator method (PM) algorithm for DOA estimation has been proposed and its performance is analyzed for the large number of antennas which constitutes massive (MIMO) antennas. The eigenvalue decomposition (EVD) of the signal's covariance matrix is an additional requirement in the previous DOA estimation algorithms which increases its computational complexity. But in the proposed algorithm it contains very low amount of computational complexity because there is no need of EVD for its autocorrelation matrix. The performance of this 2D PM algorithm is evaluated on the basis of RMSE criteria and the Monte Carlo simulated result which indicates that the proposed algorithm has much lower complexity than the ESPRIT and MUSIC algorithms as well as its performance is equivalent with the ESPRIT algorithm at the higher values of SNR.
\end{abstract}

Keywords: Direction of Arrival (DOA), Massive MIMO, Propagator Method (PM), Eigenvalue decomposition (EVD), Estimation of Signal Parameters via Rotational Invariance Techniques algorithm (ESPRIT). Multiple Signal Classification (MUSIC)

\section{Introduction}

The large array of antenna system, commonly known as massive (MIMO) is becoming an essential technology as it overcomes the ever-growing demand of high data consumption by providing higher spectral efficiency, extreme channel capacity, and link reliability. 3D Beamforming is an important technique in this regard that enables the most perfect approximation of direction of arrival (DOA), at the base radio station in order to measure the two angles, the first one is elevation angle and the second one is azimuth angle of the signal sources. In recent decade, it has been observed that the demand of mobile communication data rate is rapidly increasing and according to the predictions its growth will reach 1000 times higher in 2020. Therefore, in order to meet the ever growing demand of tremendous growth in density/volume of mobile internet traffic and connectivity, 5G will be launched in 2020[1-2].

Full-dimension (MIMO) system is one of the essential technique in the most advance generation communication system [3-4]. The addition in antenna array dimension gives the flexibility in terminal more importantly due to the spatial pre-processing at both vertical and horizontal domains. Furthermore, massive MIMO technology plays a crucial role in the latest transportation systems e.g. train carriages and high speed railway stations [5-6]. The large number of signal processing systems in today's world includes massive MIMO system and 3D beamforming for improved link reliability. 3D beamforming is a technique which enables to approximate perfectly (DOA), including both angles of the source signals [7-8]. In the previous decades DOA estimation has got much attention in the community of signal processing (SP). There are many existing methods for the DOA estimations such as maximum likelihood method, multiple signal classification (MUSIC), estimation of signal parameters via rotational invariance techniques (ESPRIT), Capon 
algorithm, parallel factor (PARAFAC) technology, etc., for the estimation of both 1D and 2D DOA [9]. However, the massive MIMO application is not yet well explored due to the high computational complexity of the dispersion matrix or covariance matrix of the input signal source. DOA estimation of 3D massive MIMO system has high complexity and limited accuracy that has made it difficult to understand and explore by researchers [10]. The previous methods of DOA has some restrictions particularly in large array of sensors, so for this purpose some fast algorithms on the basis of DOA, which have the ability of performing Eigen decomposition along with low computational load were devised such as propagator method (PM). However, this method has also some limitations such as implementation of white-Gaussian noise, further more in spatial non-uniform noise its performance criteria decreases substantially. [11-12].

Subsequently, an advance PM algorithm has been introduced in literature [13] to approximate DOA algorithms for the 1D stationary case. It has the ability of carrying out partially cross-correlation of array output data for the implementation of off-diagonal elements related to array dispersion matrix. Furthermore, the PM method has been extended to the 2D-DOA estimation in massive MIMO system for better results. As we all are well informed that propagator method is known for its low complexity method without estimating Eigen value decomposition (EVD) of the covariance matrix or dispersion matrix of the received output data, which is mentioned in the literature [14-15].

Evidently, PM algorithm is highly used in 2D-DOA estimation due to its low complexity. Therefore, we present the Propagator method to approximate 2D-DOA algorithm which is superimposed on large-scale massive MIMO system. The proposed algorithm has the advantage of calculating better angle estimation then the previous 1DDOA (PM) algorithm. The remaining sections of this paper are organized in following sections. System model is presented in second section while PM based-algorithm is introduced in third section. Moreover, forth section demonstrates the Monte Carlo simulations and performance evaluation. The paper concludes with fifth section that elaborate the findings of this paper.

Notations: Lower-case (upper-case) boldface symbols denote vectors (matrices); $(.)^{T},(.)^{H},(.)^{-1},(.)^{+}$denote the transpose, the conjugate transpose and the pseudo inverse, respectively; $I_{K}$ is the identity matrix of $K \times K$; whereas $\operatorname{diag}($.$) denotes the diagonal$ matrix and the values which are present in the brackets are diagonal elements; angle(.) presents the phase.

\section{System Model}

Figure 1 shows the structure of 2D large-scale massive MIMO system. The number of array elements are set as $M \times N$. The $\mathrm{N}$ antenna elements in the $\mathrm{X}$-axis and $\mathrm{M}$ antenna elements in the Z-axis respectively. The distance between the elements is denoted by $d$ and $d \leq \lambda / 2$, while $\lambda$ represents the wavelength of the signal. 


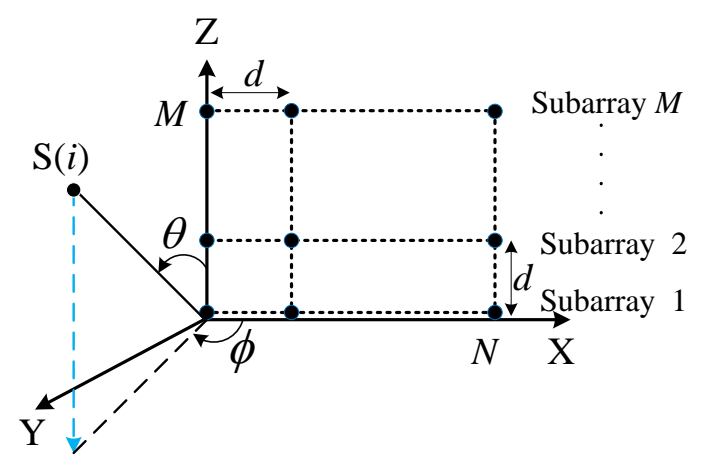

Figure 1. 2D Large-scale Massive MIMO with a Structure of $M \times N$. The DOA is Shown by $(\theta, \phi)$ which Represent Elevation Angle and Azimuth Angle Respectively

We consider there are $K$ signal sources, where $s_{i}$ represents the incident signal, $1 \leq i \leq K$ impinging on the base station (BS) and $\left(\theta_{i}, \phi_{i}\right)$ represents the elevation and azimuth angles respectively. The signal received from antenna array is modeled as:

$\mathrm{X}=\mathbf{A S}+\mathrm{N}$

$\mathbf{A}=\left[\begin{array}{ccc}\mathbf{A}_{x} & D_{1} & \mathbf{A}_{z} \\ \mathbf{A}_{x} & D_{2} & \mathbf{A}_{z} \\ \vdots & \\ \mathbf{A}_{x} & D_{M} & \mathbf{A}_{z}\end{array}\right]$

where the steering matrix at the $\mathrm{X}$-axis is:

$\mathbf{A}_{x}=\left[\begin{array}{cccc}1 & 1 & \cdots & 1 \\ e^{j 2 \pi d u_{1} / \lambda} & e^{j 2 \pi d u_{2} / \lambda} & \cdots & e^{j 2 \pi d u_{K} / \lambda} \\ \vdots & \vdots & \ddots & \vdots \\ e^{j 2 \pi d(N-1) u_{1} / \lambda} & e^{j 2 \pi d(N-1) u_{2} / \lambda} & \cdots & e^{j 2 \pi d(N-1) u_{K} / \lambda}\end{array}\right] u_{i}=\sin \theta_{i} \cos \phi_{i}$

where the steering matrix at the Z-axis array is:

$\mathbf{A}_{z}=\left[\begin{array}{cccc}1 & 1 & \cdots & 1 \\ e^{j 2 \pi d v_{1} / \lambda} & e^{j 2 \pi d v_{2} / \lambda} & \cdots & e^{j 2 \pi d v_{K} / \lambda} \\ \vdots & \vdots & \ddots & \vdots \\ e^{j 2 \pi d(\mathrm{M}-1) \mathrm{v}_{1} / \lambda} & e^{j 2 \pi d(\mathrm{M}-1) \mathrm{v}_{2} / \lambda} & \cdots & e^{j 2 \pi d(\mathrm{M}-1) \mathrm{v}_{K} / \lambda}\end{array}\right] v_{i}=\cos \theta_{i}$

where $D_{i}\left(\mathbf{A}_{z}\right)$ is the diagonal matrix with the diagonal elements being the $i^{\text {th }}$ row of the $\mathbf{A}_{z}$

$D_{i}\left(\mathbf{A}_{z}\right)=\operatorname{diag}\left\{\left(e^{j(\mathrm{i}-1) v_{1}}, e^{j(\mathrm{i}-1) v_{2}}, \cdots, e^{j(\mathrm{i}-1) v_{K}}\right)\right\}$ 
Where the transmitted signal matrix $\mathrm{S}$ is $\mathrm{S}=[\mathrm{s}(1), \mathrm{s}(2), \ldots, \mathrm{s}(\mathrm{L})] \in C^{K \times L}, \mathrm{~L}$ presents the number of snapshots, and $\mathrm{N}$ is the additive white Gaussian noise matrix, $\mathrm{N}=[\mathrm{n}(1)$, $\mathrm{n}(2), \ldots, \mathrm{n}(\mathrm{L})] \in C^{K \times L}$.

\section{Two Dimensional DOA Estimation of Low-Complexity PM Algorithm}

\subsection{Proposed Algorithm}

Matrix $\mathbf{A}$ is the combination of two subarray matrices which are describe as $\mathbf{A}_{1} \in \square^{K \times K}$ and $\mathbf{A}_{2} \in \square^{(\mathrm{MN}-K) \times K}$

$$
\mathbf{A}=\left[\begin{array}{l}
\mathbf{A}_{1} \\
\mathbf{A}_{2}
\end{array}\right]
$$

We assume (A is the full rank column matrix) and the sub matrix $\mathbf{A}_{2}$ is the linear transformation of sub matrix $\mathbf{A}_{1}$ and $\mathbf{A}_{1}$ is the non-singular matrix.

$$
\begin{aligned}
& \mathbf{A}_{2}=\mathbf{P}^{H} \mathbf{A}_{1} \\
& {\left[\mathbf{P}^{H},-\mathbf{I}_{N M-K}\right] \mathbf{A}=Q^{H} \mathbf{A}=\mathbf{O}}
\end{aligned}
$$

$\mathbf{P} \in \square^{K \times(\mathrm{MN}-K)}$ is the propagator operator and from equation (6) and (7) we have $Q^{H} \mathbf{A}=\mathbf{O}_{(\mathrm{MN}-\mathrm{K}) \times \mathrm{K}}$ Now we construct the function of spatial spectrum for the 2D-PM as

$\mathbf{P}_{2 D-P M}(\theta, \phi)=\frac{1}{\left[\mathrm{a}_{z}(\theta, \phi) \otimes \mathrm{a}_{x}(\theta, \phi)\right]^{H} Q_{0} Q_{0}^{H}\left[\mathrm{a}_{z}(\theta, \phi) \otimes \mathrm{a}_{x}(\theta, \phi)\right]}$

The covariance matrix which obtained from the received signals is shown as $\mathrm{C}=\frac{1}{L} \sum_{t=1}^{L} \mathrm{X}(\mathrm{t}) \mathrm{X}^{H}(\mathrm{t})$ for the estimation of propagator matrix $\mathbf{P}$ we partition the covariance matrix $\mathrm{C}$ as:

$\mathrm{C}=\left[\mathrm{C}_{1}, \mathrm{C}_{2}\right]$

$\mathrm{C}_{1} \in \square^{M N \times K}$ and $\mathrm{C}_{2} \in \square^{(M N-K) \times M N}$, as the noise is present the $\mathbf{P}$ estimator is:

$\hat{\mathbf{P}}=\left(\mathrm{C}_{1}^{H} C_{1}\right)^{-1} C_{1}^{H} C_{2}$

Construct matrix $\mathbf{P}_{c}$ as:

$$
\mathbf{P}_{c}=\left[\begin{array}{c}
I_{k} \\
\mathbf{P}^{H}
\end{array}\right]
$$

According to the equations (6), (7) and (12) we obtain 
$\mathbf{P}_{c} \mathbf{A}_{1}=\left[\begin{array}{l}\mathbf{A}_{1} \\ \mathbf{A}_{2}\end{array}\right]=\mathbf{A}$

We divided $\mathbf{P}_{c}$ into two sub matrices $\mathbf{P}_{a 1} \in \square^{N(\mathrm{M}-1) \times \mathrm{K}}$ and $\mathbf{P}_{a 2} \in \square^{N(\mathrm{M}-1) \times \mathrm{K}}$. $\mathbf{P}_{a 1}$ belongs to the first $N(M-1)$ rows of $\mathbf{P}_{c}$, while $\mathbf{P}_{a 2}$ belongs to the last $N(M-1)$ rows of $\mathbf{P}_{c}$. We also use $\mathbf{A}_{a}$ and $\mathbf{A}_{b}$ to represent the first $N(M-1)$ rows and the last $N(M-1)$ rows of $\mathbf{A}$.

$\left[\begin{array}{l}\mathbf{P}_{a 1} \\ \mathbf{P}_{a 2}\end{array}\right] \mathbf{A}_{1}=\left[\begin{array}{l}\mathbf{A}_{a} \\ \mathbf{A}_{b}\end{array}\right]=\left[\begin{array}{c}\mathbf{A}_{a} \\ \mathbf{A}_{a} \Phi_{z}\end{array}\right]$

In the above equation, $\boldsymbol{\Phi}_{z}$ is for the diagonal matrices of $D_{2}$ and $\mathbf{A}_{z}=\operatorname{diag}\left\{\left(\mathrm{e}^{j v_{1}}, \mathrm{e}^{j v_{2}}, \ldots \ldots . . \mathrm{e}^{j v_{K}}\right)\right\}$

Therefore

$\mathbf{P}_{a 1}^{+} \mathbf{P}_{a 2}=\mathbf{A}_{1} \boldsymbol{\Phi}_{z} \mathbf{A}_{1}^{-1}$

According to the definition $\boldsymbol{\Psi}_{z}$ must be equal to the

$\boldsymbol{\Psi}_{z}=\mathbf{P}_{a 1}^{+} \mathbf{P}_{a 2}$

The eigenvalues of $\boldsymbol{\Psi}_{z}$ and $\boldsymbol{\Phi}_{z}$ are same and $\mathbf{A}_{1}$ is the eigenvectors matrix of $\boldsymbol{\Phi}_{z}$.

We perform the eigen-decomposition of $\boldsymbol{\Psi}_{z}$ and the estimator of can be obtained by $v_{k}$.

$\hat{v}_{k}=\frac{\operatorname{angle}\left(\lambda_{k}\right) \lambda}{2 \pi d}$

where $\lambda_{k}=e^{j 2 \pi d v_{k} / \lambda}$ is the $K^{\text {th }}$ eigenvalue of $\boldsymbol{\Psi}_{x}, k=1,2, \cdots, K$.

By equation (13) we have

$\mathbf{P}_{c}=\left[\begin{array}{ccc}\mathbf{A}_{x} & D_{1} & \mathbf{A}_{z} \\ \mathbf{A}_{x} & D_{2} & \mathbf{A}_{z} \\ \vdots & \\ \mathbf{A}_{x} & D_{M} & \mathbf{A}_{z}\end{array}\right] \mathbf{A}_{1}^{-1}$

We recreate $\mathbf{P}_{c}$ to $\mathbf{P}_{c}^{\prime}$

$$
\mathbf{P}_{c}^{\prime}=\mathbf{P}_{c} \mathbf{A}_{x 1}
$$

By putting the values of (18) in (19), we get 


$$
\mathbf{P}_{c}^{\prime}=\left[\begin{array}{ccc}
\mathbf{A}_{x} & D_{1} & \mathbf{A}_{z} \\
\mathbf{A}_{x} & D_{2} & \mathbf{A}_{z} \\
\vdots & \\
\mathbf{A}_{x} & D_{M} & \mathbf{A}_{z}
\end{array}\right] \mathbf{A}_{1}^{-1}
$$

Similarly, we have to fragment $\mathbf{P}_{c}^{\prime}$ into $\mathbf{P}_{a 1}^{\prime}$ and $\mathbf{P}_{a 2}^{\prime}$ for the presentation of first $N(M-1)$ rows and the last $N(M-1)$ rows, respectively.

$$
\boldsymbol{\Psi}_{x}=\mathbf{P}_{a 1}^{\prime+} \mathbf{P}_{a 2}^{\prime}
$$

According to the theoretical point of view, $\boldsymbol{\Psi}_{z}$ and $\boldsymbol{\Psi}_{x}$ have the same eigenvectors matrix that is.

$$
\boldsymbol{\Psi}_{x}=\mathbf{A}_{1} \boldsymbol{\Phi}_{x} \mathbf{A}_{1}^{-1}
$$

The estimator of $u_{k}$ can be calculated by

$\hat{u}_{k}=\frac{\operatorname{angle}\left(\varepsilon_{k}\right) \lambda}{2 \pi d}$

where $\varepsilon_{k}=e^{j 2 \pi d u_{k} / \lambda}$ is the $K^{\text {th }}$ eigenvalue of $\boldsymbol{\Psi}_{x}$ and $k=1,2, \cdots, K$. Finally, the correct paired of the elevation and azimuth angle $\left(\widehat{\theta}_{k}, \widehat{\phi}_{k}\right)$ can be projected through following expressions.

$$
\begin{aligned}
& \hat{\theta}_{k}=\cos ^{-1}\left(\hat{\mathrm{v}}_{k}\right) \\
& \hat{\phi}_{k}=\cos ^{-1}\left(\frac{\hat{u}}{\sqrt{1-\hat{v}_{k}^{2}}}\right)
\end{aligned}
$$

\subsection{Two-Dimensional MUSIC Algorithm}

The MUSIC algorithm is attractive for its accuracy and immunity to noise due to the use of the signal subspaces of the receive signals at the antenna array. A brief introduction of 2-D MUSIC algorithm is presented. It simultaneously estimates the azimuthal angle and the elevation angle of multiple signal sources. 2D-MUSIC is similar to the onedimensional case, but the direction is given as

$$
\mathbf{A}=\left[\begin{array}{c}
\mathbf{A}_{\mathbf{x}} D_{1}\left(\mathbf{A}_{z}\right) \\
\mathbf{A}_{\mathbf{x}} D_{2}\left(\mathbf{A}_{z}\right) \\
\vdots \\
\mathbf{A}_{\mathbf{x}} D_{M}\left(\mathbf{A}_{z}\right)
\end{array}\right]
$$

Another form is

$$
\mathbf{A}=\left[\mathbf{a}_{z}\left(\theta_{1}, \phi_{1}\right) \otimes \mathbf{a}_{\mathbf{x}}\left(\theta_{1}, \phi_{1}\right), \mathbf{a}_{z}\left(\theta_{2}, \phi_{2}\right) \otimes \mathbf{a}_{\mathbf{x}}\left(\theta_{2}, \phi_{2}\right), \cdots, \mathbf{a}_{z}\left(\theta_{K}, \phi_{K}\right) \otimes \mathbf{a}_{\mathbf{x}}\left(\theta_{K}, \phi_{K}\right)\right]
$$


In the above equation $\mathbf{a}_{x}\left(\theta_{k}, \phi_{k}\right), \mathbf{a}_{z}\left(\theta_{k}, \phi_{k}\right), k=1, \ldots, K, \mathbf{A}_{x}, \mathbf{A}_{z}$, the first $\boldsymbol{k}$ column vector. The space spectrum function of the 2D-MUSIC algorithm is written as under

$$
P_{2 D-M U S I C}(\theta, \phi)=\frac{1}{\left[\mathbf{a}_{z}(\theta, \phi) \otimes \mathbf{a}_{\mathbf{x}}(\theta, \phi)\right]^{H} \mathbf{V}_{\mathbf{N}} \mathbf{V}_{\mathbf{N}}{ }^{H}\left[\mathbf{a}_{z}(\theta, \phi) \otimes \mathbf{a}_{\mathbf{x}}(\theta, \phi)\right]}
$$

In the above equation $\mathbf{V}_{\mathbf{N}}$ is the matrix whose columns are the noise subspace eigenvectors.

\subsection{Complexity Analysis}

As we early declared that the proposed algorithm has much lower complexity than the estimation of signal parameters via rotational invariance technique (ESPRIT) algorithm and the multiple signal classification (MUSIC) algorithm on the basis of complexity analysis, in which $N \times M$ represents the number of antenna elements which lies in the dimensions of the $\mathrm{X}$-axis and $\mathrm{Z}$-axis respectively. Furthermore ' $\mathrm{L}$ ' is use to represent number of snapshots while the signal sources are represented by ' $K$ '. Now here we present the complexity of algorithms.

$O\left(L M^{2} N^{2}+M N K^{2}+M N(M N-K)+4 K^{3}+K^{2}(M N-K)+2 K^{2}(M-1) N+K^{2} M N+(N\right.$

$-1) M K$ is the complexity of proposed algorithm. Now the complexity of the ESPRIT algorithm is as $O\left(L M^{2} N^{2}+M^{3} N^{3}+2 K^{2}(M-1) N 3 K^{3}+K^{2} M N+(N-1) M K\right) K^{2} M N+$ $(N-1) M K)$. Now the complexity of MUSIC is $O\left\{L M^{2} N^{2}+M^{3} N^{3}+n_{g}[M N(M N-K)+\right.$ $M N-K]$ \} among them $n_{g}$ is the number of global search.

Figure. 2 clearly shows the comparison based on complexity between the algorithms along with the properties of $N=M, \mathrm{~K}=6$ and, $\mathrm{L}=100$. The figure proves that ESPRIT and MUSIC algorithm has higher computation complexity than the proposed algorithm which is 2D-PM. And figure also clarifies that rise of computational load in the proposed algorithm is much lower as compared to the computational load of MUSIC and ESPRIT algorithm.

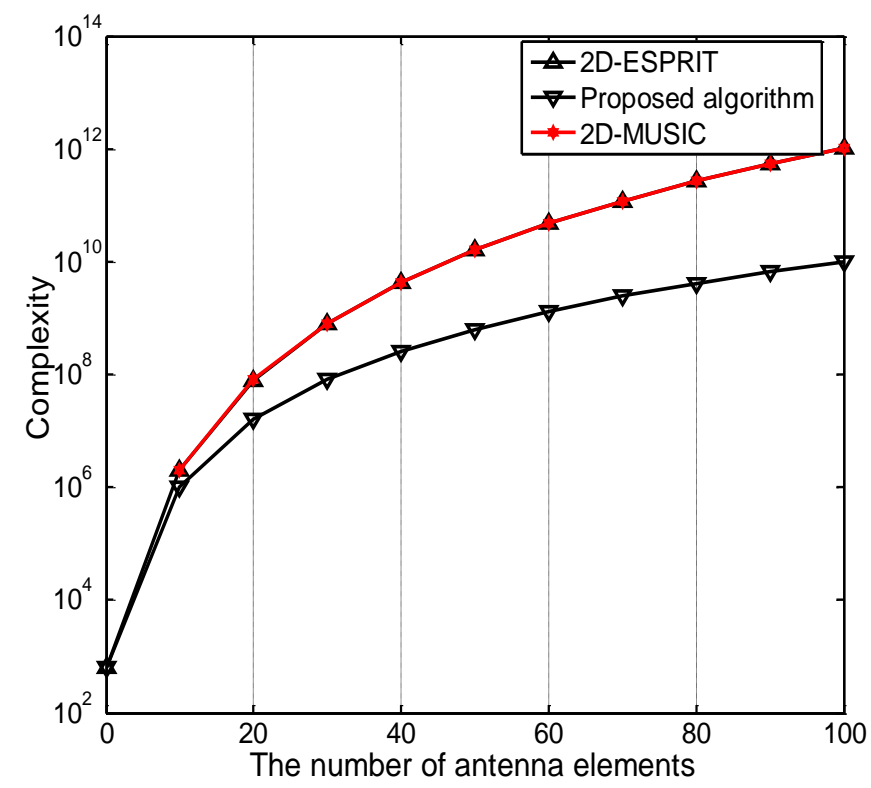

Figure 2. Algorithms Complexity Comparison 


\subsection{Advantages of the Proposed Algorithm}

- In proposed algorithm, we discover automatic matching of the angle parameters; in addition it avoids the extra computational burden which has been brought by the matching of angle parameters.

- While comparing it with the performance of ESPRIT algorithm, we discover that the signal's covariance matrix doesn't require any eigenvalue decomposition.

- The performance of the proposed algorithm reaches to the performance of the ESPRIT algorithm when the SNR proceeds towards the higher values.

\section{Simulation and Analysis}

The angle estimation of 2D PM algorithm has been carried out by using 1000 simulations of Monte Carlo. The preferable technique to measure the DOA accurately is the root means square error (RMSE) which can be define as:

$R M S E=\frac{1}{K} \sum_{k=1}^{K} \sqrt{\frac{1}{1000} \sum_{l=1}^{1000}\left[\left(\hat{\theta}_{k, n}-\theta_{k}\right)^{2}\left(\hat{\phi}_{k, n}-\phi_{k}\right)^{2}\right]}$

Where $\hat{\theta}_{k, n}$ is the estimation of elevation angle $\theta_{k}$ at the $n^{\text {th }}$ Monte Carlo trial and similarly for $\hat{\phi}_{k, n}$. There are 9 incident to narrow band signals which are shown in Fig. 1 and their direction of arrival is given as:

$\left(25^{\circ}, 20^{\circ}\right),\left(35^{\circ}, 60^{\circ}\right),\left(40^{\circ}, 110^{\circ}\right),\left(80^{\circ}, 25^{\circ}\right),\left(85^{\circ}, 65^{\circ}\right),\left(90^{\circ}, 115^{\circ}\right),\left(130^{\circ}, 30^{\circ}\right)$, $\left(135^{\circ}, 70^{\circ}\right),\left(140^{\circ}, 120^{\circ}\right)$.

The spacing between the array element is $d=\lambda / 2$. Where $N$ and $M$ are the number of sensors at the $\mathrm{X}$-axis and $\mathrm{Z}$-axis respectively. The number source target represented by $K$ and $L$ represents the number of snapshots.

\subsection{Performance Evaluation}

In this section the numerical conclusions demonstrate the performance criteria of the proposed algorithm, which consists of simulation trails up to 1000 as discussed earlier. The space assigned to the adjacent antenna elements is $d=0.5 \lambda$. The number of antennas are installed in two dimensions which is $\mathrm{M}=\mathrm{N}=16$. Snapshots are denoted by $\mathrm{L}$ and they are equal to 100. For elevation and azimuth angles there are nine narrow band signal sources that is applied to the antenna array which are:

$\theta=\left\{25^{\circ}, 35^{\circ}, 40^{\circ}, 80^{\circ}, 85^{\circ}, 90^{\circ}, 130^{\circ}, 135^{\circ}, 140^{\circ}\right\}, \phi=\left\{20^{\circ}, 60^{\circ}, 110^{\circ}, 25^{\circ}, 65^{\circ}, 115^{\circ}, 30^{\circ}, 70^{\circ}\right.$, $\left.120^{\circ}\right\}$

The different SNRs are presented in Figure. 3 and Figure. 4, which demonstrate the DOA estimation of the proposed algorithm. The simulation results point out the performance of the DOA estimation is poor in low SNR conditions but in high SNR the results are totally different, that estimation of the desired elevation and azimuth angles are highly accurate.

The number of signal sources are denoted by $K=10$. The evaluation of elevation and azimuth angles under root means square error (RMSE) implemented for the different number of sources signals are presented in Figure. 5. It is the clear condition for massive MIMO which is $\mathrm{K}<<\mathrm{M}$ and by this condition it clearly proves that if we increase the number of signal sources then the performance of DOA estimation becomes worse.

Figure. 6 indicates the RMSE of the proposed algorithm in different number of snapshots. As the number of snapshots increases then the DOA estimation performance becomes better. This happens due to the increase in the number of snapshots which acquires more and more accurate covariance matrix. 
In Figure. 7, the algorithm is used to compare the different values of antenna elements. As can be seen from the figure, the algorithm performance approaches towards better outcomes with increase in the number of array elements, the simulation indicates that as we increase the number of antenna in the massive MIMO the angle estimation becomes more accurate, because it increases the diversity gain.

In Figure. 8, a comparison of proposed, 2D ESPRIT and, 2D-MUSIC algorithms is shown. The figure illustrates that the proposed algorithm acquires a high RMSE in low SNR but the performance reaches to ESPRIT algorithm when the SNR moves towards higher values. Therefore, the proposed algorithm has wide range of applications on the basis of DOA estimation accuracy.

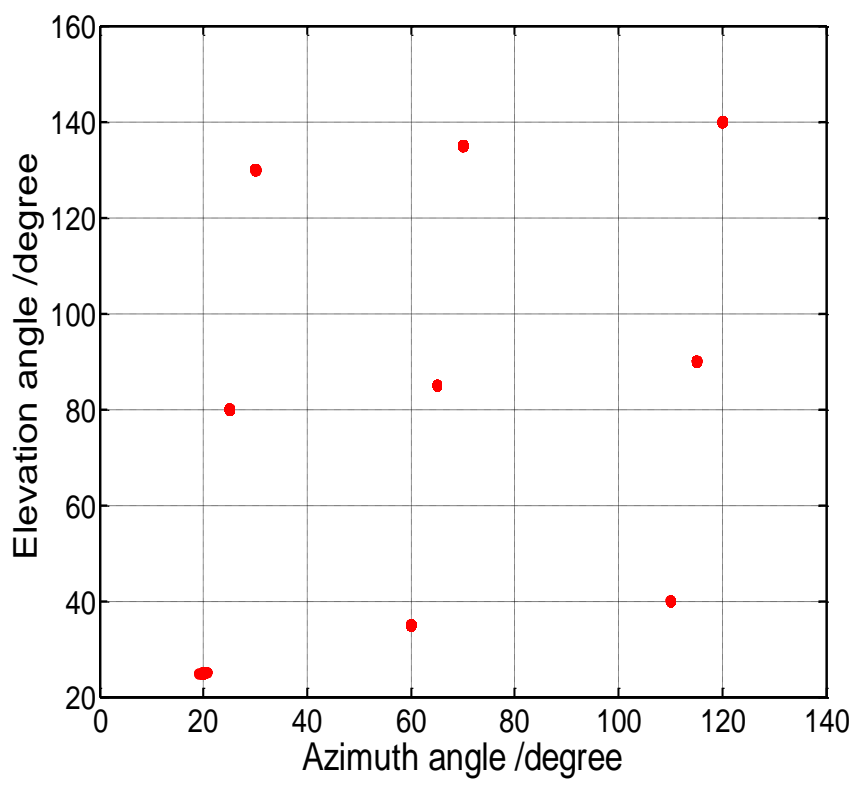

Figure 3. Angle Estimation Performance $(M=N=16, L=100, S N R=15 d B)$

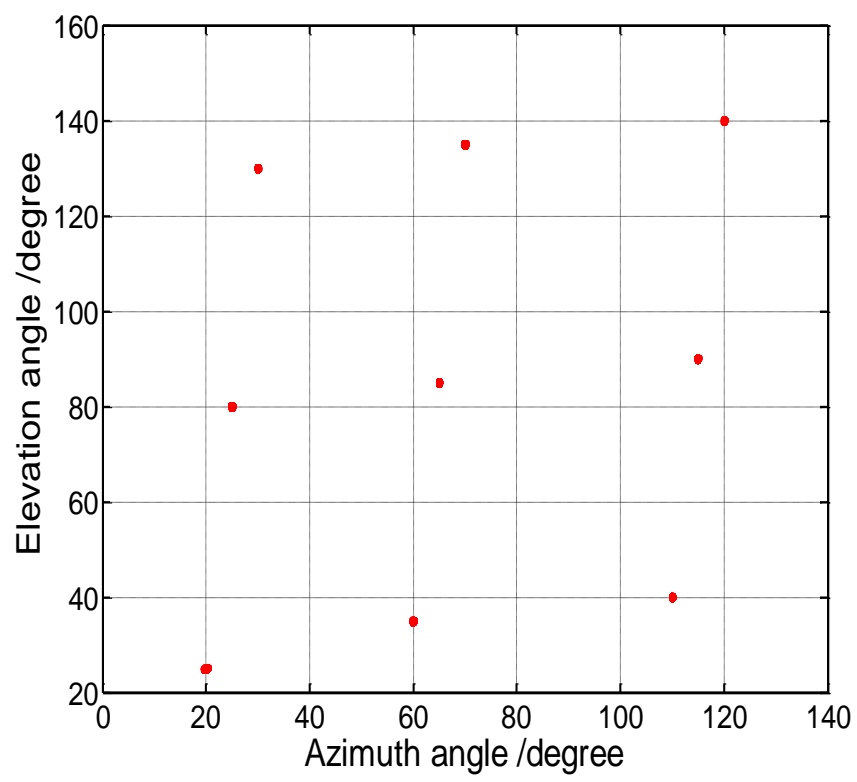

Figure 4. Angle Estimation Performance $(M=N=16, L 100, S N R=20 d B)$ 


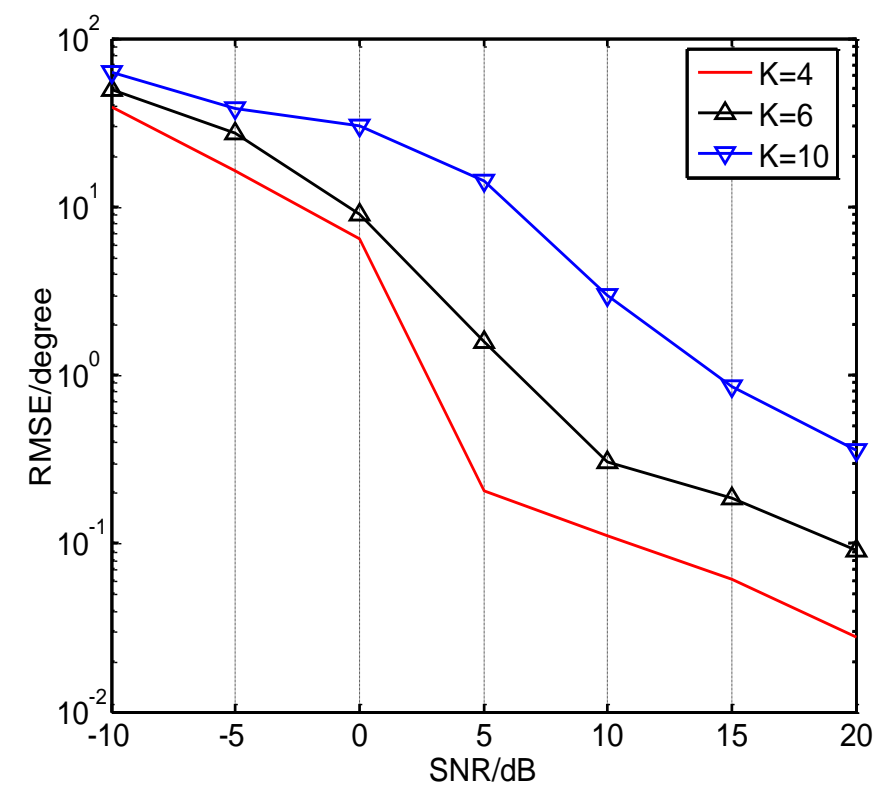

Figure 5. RMSE Estimation Performance with Different Numbers of Source Signals $(M=N=16, L=100)$

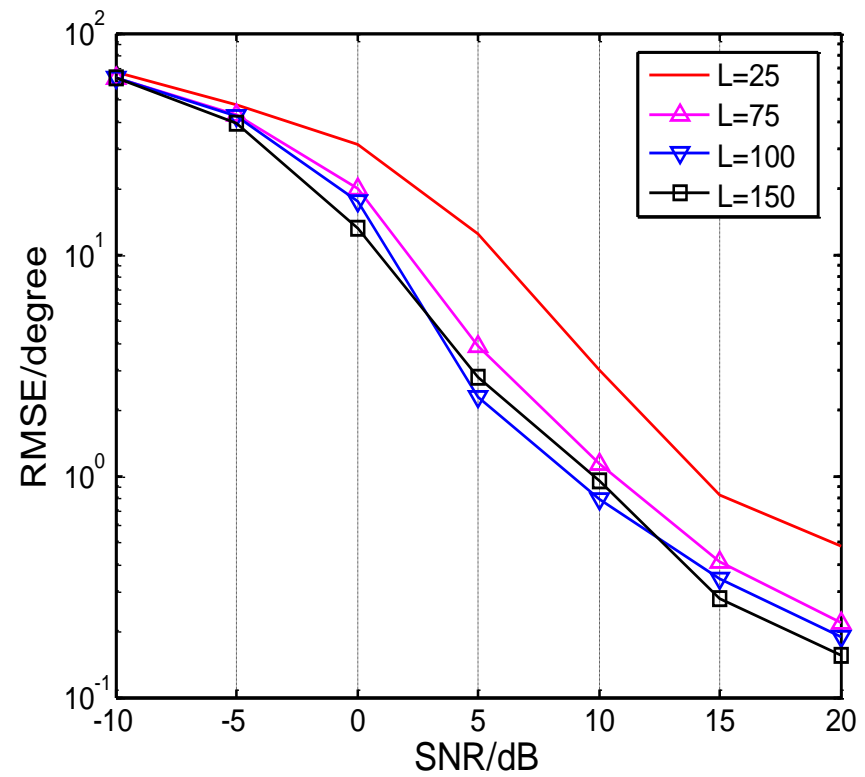

Figure 6. RMSE Estimation Performance in Different Numbers of Snapshots $(M=N=16, K=6)$ 


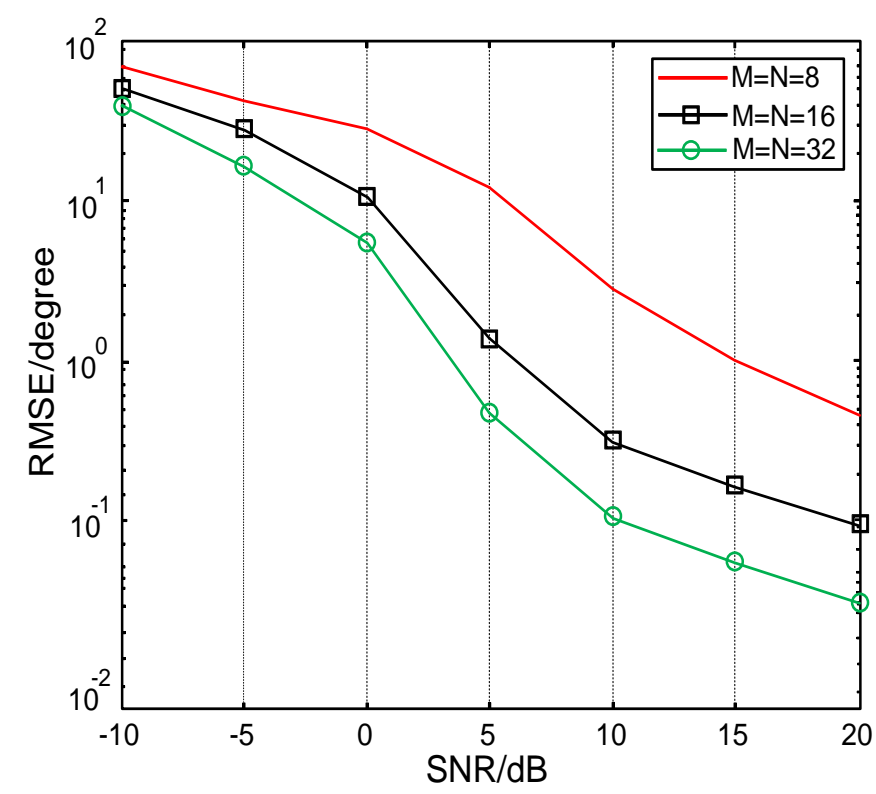

Figure 7. Angle Estimation Performance under Different Number of Sources $(K=6, L=100)$

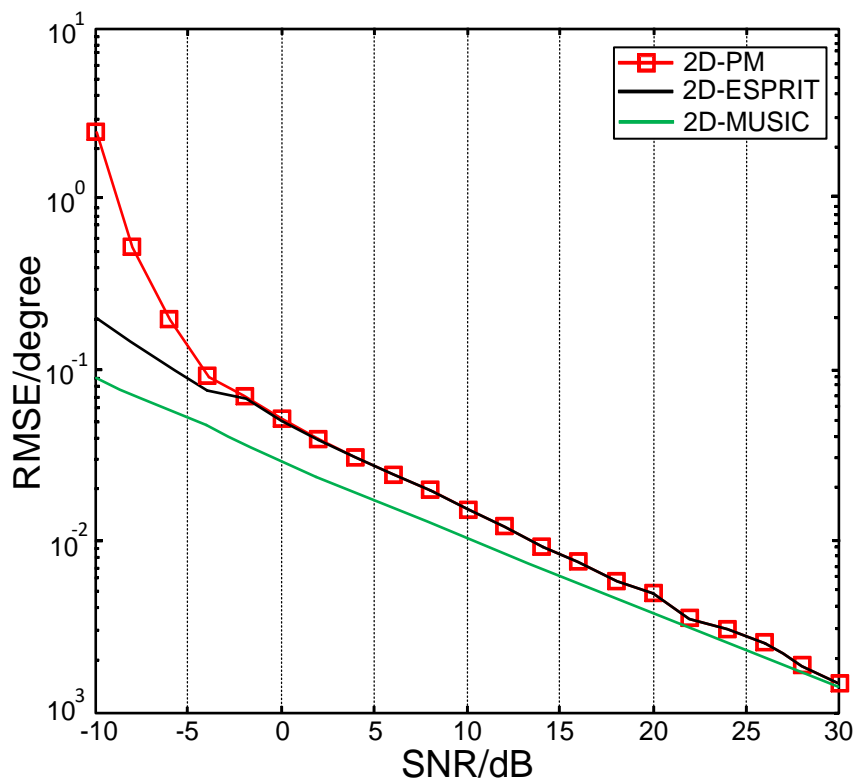

Figure 8. RMSE Performance of Three Algorithms

\section{Conclusion}

In this paper, a low-complexity 2D-DOA based on PM algorithm for the Massive MIMO system using 2D antenna array has been proposed. The proposed algorithm avoids EVD for the signal's covariance matrix. Moreover, there is no need of peak searching thus it is simple in its structure. The proposed algorithm acquires a high RMSE in low SNR but the performance approaches to ESPRIT algorithm when the SNR approaches to higher values. The simulation results verify our analytical approach. Therefore, the proposed algorithm has wide range of applications on the basis of DOA estimation accuracy. 


\section{References}

[1] J. G. Andrews et al., “what will 5G be,’ IEEE J. Sel. Areas Commun., vol. 32, no. 6, (2014), pp. 10651082.

[2] A. Osseiran et al., "Scenarios for 5G mobile and wireless communications: The vision of the METIS project,'” I IEEE Commun. Mag., vol. 52, no. 5, (2014), pp. 26-35.

[3] E. Larsson, O. Edfors, F. Tufvesson, and T. Marzetta, "Massive MIMO for next generation wireless systems,'” IEEE Commun. Mag., vol. 52, no. 2, (2014), pp. 186-195. EEE Commun. Mag., vol. 52, no. 5, May (2014), pp. 26-35.

[4] L. Lu, G. Y. Li, A. L. Swindlehurst, A. Ashikhmin, and R. Zhang, "An overview of massive MIMO: Benefits and challenges,"' IEEE J. Sel. Topics Signal Process., vol. 8, no. 5, (2014), pp. 742-758.

[5] B. Ai et al., "Challenges toward wireless communications for high-speed railway," IEEE Trans. Intell. Transp. Syst., vol. 15, no. 5, (2014), pp. 2143-2158.

[6] B. Ai et al., "Future railway services-oriented mobile communications network," IEEE Commun. Mag., vol. 53, no. 10, (2015), pp. 78-85.

[7] "Future technology trends of terrestrial IMT systems," ITU, Geneva, Switzerland, Tech. Rep. ITU-R M.2320-0, (2014).

[8] H. Halbauer, S. Saur, J. Koppenborg, and C. Hoek, "3D beamforming: Performance improvement for cellular networks,'’ Bell Labs Tech. J., vol. 18, no. 2, (2013), pp. 37-56.

[9] H. Krim and M. Viberg, "Two decades of array signal processing research: The parametric approach," IEEE Signal Process. Mag., vol. 13, no. 4, (1996), pp. 67-94.

[10] X. Cheng et al., "Communicating in the real world: 3D MIMO," IEEE Wireless Commun., vol. 21, no. 4, (2014), pp. 136-144.

[11] J. Munier and G. Y. Delisle, "Spatial analysis using new properties of the cross-spectral matrix", IEEE Trans. Sig. Proces., vol. 39, no. 3, (1991), pp. 746-749.

[12] M. Frikel, "Localization of sources radiating on a large antenna", Proceedings of the 13th international Conference of EUSIPCO Eur. Signal Processing., Antalya, Turkey, (2005).

[13] J. Chen, Y. Wu, H. Cao, and H. Wang, "Fast algorithm for DOA estimation with partial covariance matrix and without Eigen decomposition", J. Sig. Inform. Process. vol. 2, no. 4, (2011), pp. 266-259.

[14] S. Marcos, A. Marsal, and M. Benidir, "Te propagator method for source bearing estimation," Signal Processing, vol. 42, no. 2, (1995), pp. 121-138.

[15] Liu L, Li Y, Zhang J, "DOA estimation, and achievable rate analysis for 3D millimeter wave massive MIMO systems," Signal Processing Advances in Wireless Communications (SPAWC), 2014 IEEE 15th International Workshop on. IEEE, (2014), pp. 6-10.

[16] Wang A, Liu L, Zhang J, "Low complexity direction of arrival (DOA) estimation for 2D massive MIMO systems," IEEE Globecom Workshops, (2012), pp.703 - 707.

[17] Yang, Kai Yu, J. Y. Wu, and W. H. Li, "A low-complexity direction-of-arrival estimation algorithm for full-dimension massive MIMO systems," Communication Systems (ICCS), 2014 IEEE International Conference on. IEEE, (2014).

[18] $\mathrm{Hu}$, Anzhong, et al, "An ESPRIT-based approach for 2-D localization of incoherently distributed sources in massive MIMO systems." IEEE Journal of Selected Topics in Signal Processing 8.5, (2014), pp.996 - 1011.

[19] [19] K.-Y. Yang, J.-Y. Wu, and W.-H. Li, "A low-complexity direction-ofarrival estimation algorithm for full-dimension massive MIMO systems," in Communication Systems (ICCS), 2014 IEEE International Conference on. IEEE, (2014), pp. 472-476.

[20] C. Jian, S. Wang, and L. Lin, "Two-dimensional DOA estimation of coherent signals based on 2D unitary ESPRIT method," in Signal Processing, 2006 8th International Conference on, vol. 1. IEEE, (2006).

[21] $\mathrm{Hu}$, Anzhong, et al, "An ESPRIT-based approach for 2-D localization of incoherently distributed sources in massive MIMO systems." IEEE Journal of Selected Topics in Signal Processing 8.5, (2014), pp.996 - 1011.

[22] Huipeng Meng; Zhi Zheng; Yuxuan Yang; Kehong Liu; Yan Ge. "A low-complexity 2-D DOA estimation algorithm for massive MIMO systems." 2016 IEEE/CIC International Conference on Communications in China (ICCC), (2016), pp.1-5 